\title{
Postpartum Sudden Cardiac Death After Spontaneous Coronary Artery Dissection in a Patient With Fibromuscular Dysplasia
}

\author{
Tannaz Shoja ${ }^{\mathrm{a}, \mathrm{c}}$, Craig Basman ${ }^{\mathrm{b}}$, Suresh Jain ${ }^{\mathrm{a}, \mathrm{b}}$, Aditya Mangla ${ }^{\mathrm{a}, \mathrm{b}}$, \\ Zoran Lasic ${ }^{\mathrm{a}, \mathrm{b}}$
}

\begin{abstract}
Spontaneous coronary artery dissection (SCAD) is a rare entity that can cause acute myocardial infarction and sudden cardiac death (SCD) which often goes unrecognized. We report a case of SCAD in a young postpartum female who presented with sudden cardiac arrest. The patient was managed medically and found to have fibromuscular dysplasia (FMD). After being stabilized in the critical care unit, coronary angiography was performed which showed dissection of the left main artery, intramural hematoma, and the culprit lesion. Further investigation showed dissection of the left vertebral artery which was all consistent with a diagnosis of FMD. The patient was followed as an outpatient and a repeated coronary angiography demonstrated healed dissection site. In conclusion, this case exemplifies that prompt diagnosis along with medical management without the need of coronary artery bypass graft, and percutaneous coronary intervention can improve survival in SCAD.
\end{abstract}

Keywords: Spontaneous coronary artery dissection; Cardiac arrest; Fibromuscular dysplasia; Postpartum

\section{Introduction}

We report a case of spontaneous coronary artery dissection (SCAD) presenting as sudden cardiac death (SCD) in a 38-year-old, 2 weeks postpartum female. Cardiac catheterization revealed an ulcerated plaque with suspicion for intramural hematoma in the mid-segment of the left main coronary artery, consistent with SCAD. This case poses several interesting clinical decision-making obstacles, including management of

Manuscript submitted September 4, 2017, accepted September 12, 2017

a Jamaica Hospital Medical Center, 8900 Van Wyck Expy, Richmond Hill, NY 11418, USA

bLenox Hill Hospital Medical Center, 100 E 77th St, New York, NY 10075, USA

${ }^{\mathrm{c} C o r r e s p o n d i n g ~ A u t h o r: ~ T a n n a z ~ S h o j a, ~ J a m a i c a ~ H o s p i t a l ~ M e d i c a l ~ C e n t e r, ~} 8900$ Van Wyck Expy, Richmond Hill, NY 11418, USA.

Email: tannazshoja@gmail.com

doi: https://doi.org/10.14740/cr587w left main SCAD with a large minimal lumen cross-sectional area (CSA) on intravascular ultrasound (IVUS) in true lumen, further diagnostic workup for fibromuscular dysplasia (FMD) and the need for implantable cardioverter defibrillator (ICD) implantation in patients that present with SCD in presence of SCAD.

\section{Case Report}

A 38-year-old female 2 weeks postpartum from an uncomplicated fourth pregnancy presented to the emergency room with cardiac arrest. The arrest occurred while the patient was breastfeeding her newborn and was witnessed by her husband. Cardiopulmonary resuscitation was immediately initiated by her husband, and was found to be in ventricular-tachycardia when the EMS arrived. She was defibrillated several times, intubated in the field and regained a pulse on arrival to the emergency room.

On arrival, the patient was hypertensive (BP 155/85 mm $\mathrm{Hg}$ ), tachycardic (HR 79/min), and tachypnic (RR 21/min) on constant manual ventilation with oxygen saturation of $97 \%$ on $\mathrm{FiO}_{2} 100 \%$. On neurologic examination, she opened her eyes in response to noxious stimuli, pupils were equal, round, and reactive to light, gag reflex was present and she was breathing over the ventilator. Her cardiac examination revealed normal heart sound, regular rhythm without appreciable rubs or murmurs with an otherwise unremarkable physical exam. Her electrocardiogram revealed normal sinus rhythm with non-specific ST changes. Her initial troponin level was 3.3 which peaked to $61 \mathrm{ng} / \mathrm{mL}$. Transthoracic echocardiogram revealed moderate left ventricular dysfunction with ejection fraction of $40 \%$, with anterior hypokinesis and without valvular abnormalities. Left heart catheterization revealed a large size left main coronary artery with a $50 \%$ irregular contour, eccentric, and ulcerated plaque and 70\% mid-left anterior descending (LAD) tubular stenosis with thrombolysis in myocardial infarction (TIMI) 3 flow (Fig. 1). Medical management with beta blockade, dual antiplatelet therapy (aspirin/clopidogrel) and heparin was initiated. Magnetic resonance angiography (MRA) of the head and neck as well as abdominal arteries was performed to evaluate for the presence of other vascular involvement related to FMD. There were focal regions of dissection in the left vertebral artery with a high index of suspicion for FMD. The patient 


\section{9/8/16 Cath}

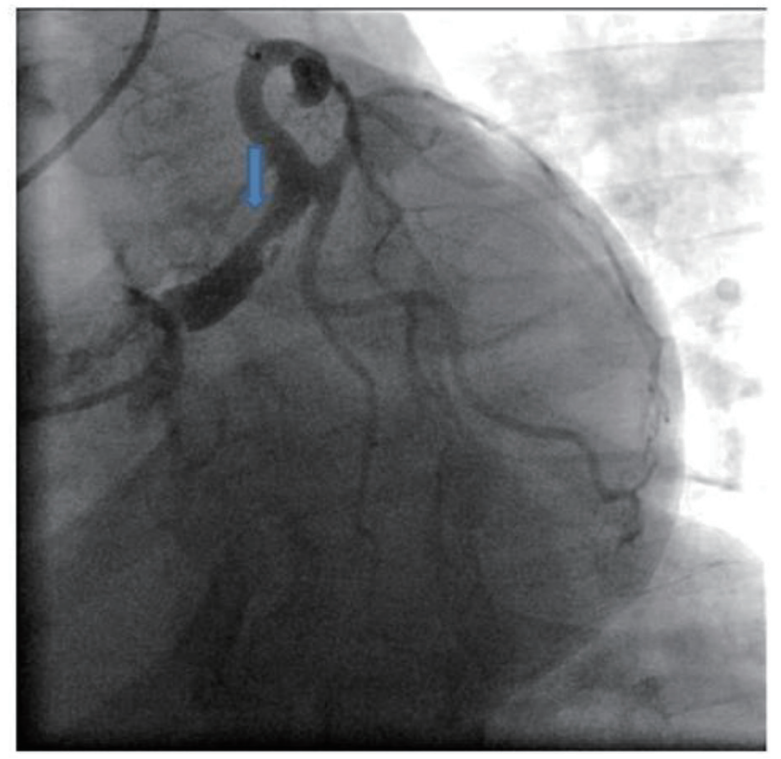

Figure 1. Left anterior oblique caudal view revealing a large sized left main artery with a $50 \%$ irregular contoured, eccentric and ulcerated plaque.

remained stable and was extubated 3 days after admission. A repeat angiography (5 days after initial) revealed improved angiographic appearance of her left main and LAD (Fig. 2). The IVUS revealed significant intramural hematoma and a left main artery lumen CSA of $8.1 \mathrm{~mm}^{2}$ (Fig. 3). The stenotic area of the mid-LAD was also consistent with SCAD.

The patient was treated with guideline directed medical therapy because CSA was $>6 \mathrm{~mm}^{2}$, TIMI 3 flow, absence of

\section{9/13/16 Cath}

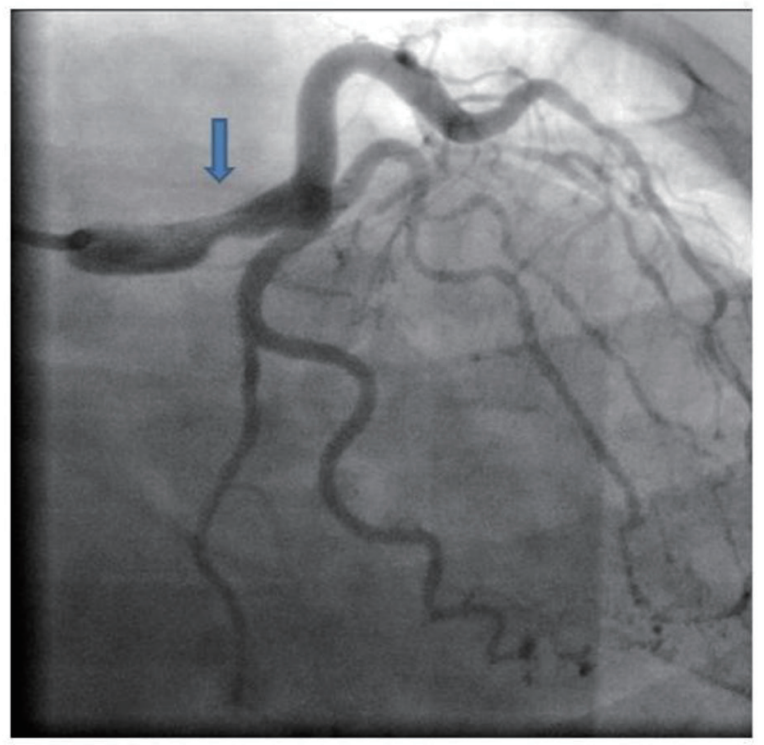

Figure 2. A repeat angiography revealing improved angiographic appearance of her left main.

hemodynamic instability, ischemia or arrhythmias at the time of diagnosis. The patient responded well to medical therapy and a repeat catheterization 3 months later demonstrated improved angiographic appearance. The vertebral artery dissection found on the MRA supported the diagnosis of FMD, while renal, splenic iliac and hepatic arteries were normal.

The patient was discharged home with a LifeVest wearable defibrillator on guideline directed medical therapy and was asymptomatic with no events at 3-month follow-up. A repeated

\section{Proximal left main \\ Intramural hematoma \\ Distal left main}

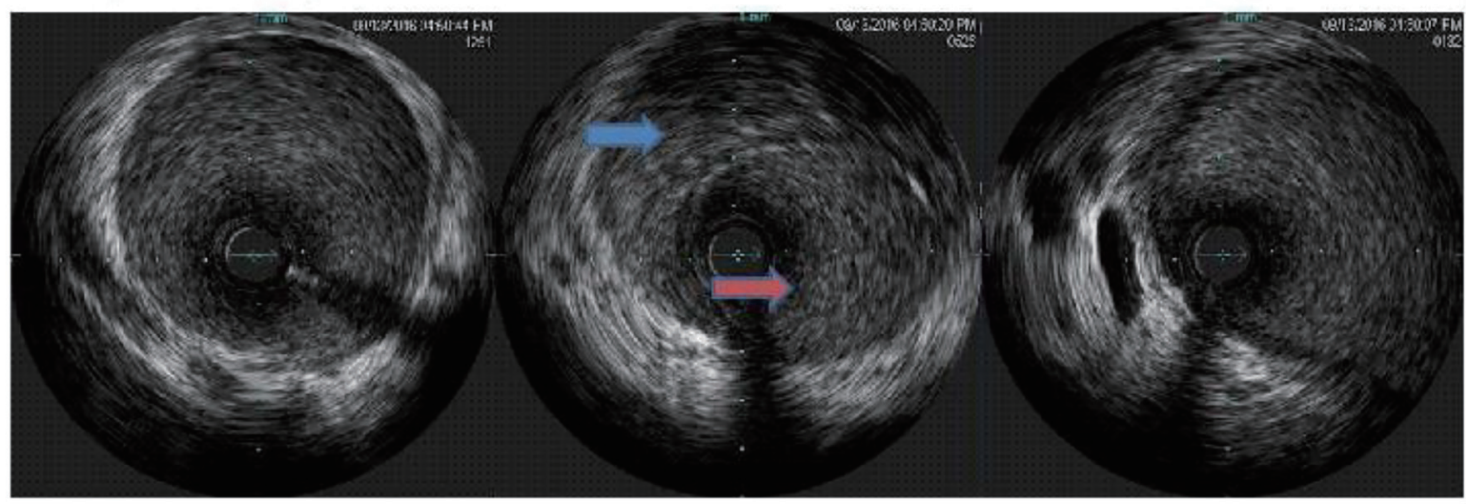

Blue arrow: false lumen

Red arrow: true lumen

Figure 3. The intravascular ultrasound (IVUS) revealing significant intramural hematoma and a left main artery lumen cross sectional area (CSA). 


\section{$12 / 22 / 16$}

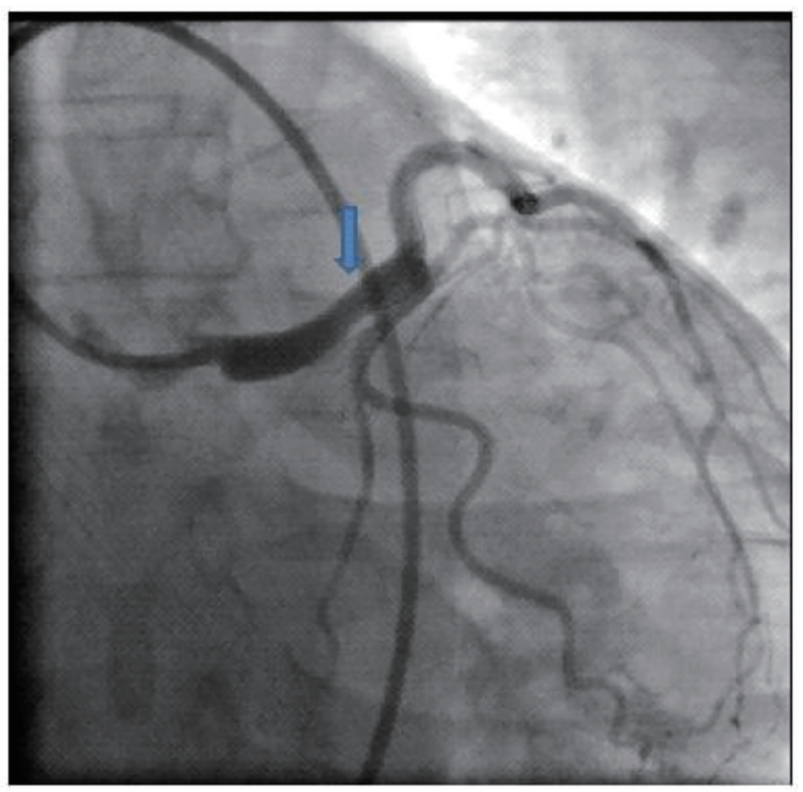

Figure 4. Repeat cardiac catheterization showing improved left main lesion (blue arrow).

angiography revealed continuous angiographic improvement of her left main artery lesion site (Fig. 4), while the IVUS revealed complete resolution of the intramural hematoma (Fig. $5)$.

\section{Discussion}

The management of SCAD is controversial, since no clinical trial has compared medical therapy against revascularization strategies. Standard medical therapy of acute coronary syndrome (ACS) has not been particularly studied for SCAD [1]. Beta blockers theoretically reduce coronary arterial wall pressure, ventricular arrhythmias, and long-term survival; hence they are being administered usually in acute and long-term management of SCAD [1,2]. Since SCAD is often healed spontaneously, conservative management has been preferred if possible [3-10]. Revascularization is considered for patients who are hemodynamically unstable, have ventricular arrhythmias, unstable lesions in major coronary arteries and unresolved/ongoing ischemia [11]. These cases may be managed by percutaneous coronary intervention (PCI) or coronary artery bypass grafting (CABG), depending on the coronary anatomy [12].

This case posed challenges in our clinical decisions making. Foremost issue was the acute lesion management in presence of left main artery involvement. Options included revascularization with PCI or CABG as opposed to medical therapy [12]. We opted for medical therapy because of the patient's absence of hemodynamic instability, arrhythmia, or ongoing ischemia, with TIMI 3 flow and a CSA in true lumen on IVUS of $8.2 \mathrm{~mm}^{2}$. Furthermore, PCI in cases of intramural hematoma could lead to propagation of dissection (intramural hematoma) proximal and distal along the coronary tree requiring extensive stenting and compromising flow [13].

The second dilemma was secondary prevention of SCD in patients with cardiac arrest. The patient's SCD was consequence of SCAD presenting as ACS. There are no randomized clinical trials comparing medical therapy and ICD in patients with SCAD and SCD [14]. We elected to use a wearable cardioverter defibrillator and monitor the patient. She had no events over the next 3 months, with normalization of left ventricular ejection fraction and healing of intramural hematoma on follow-up with IVUS. The wearable cardioverter defibrillator was discontinued after 3 months and no ICD was implanted. The patient remained on anti-

\section{Pre-lesion}

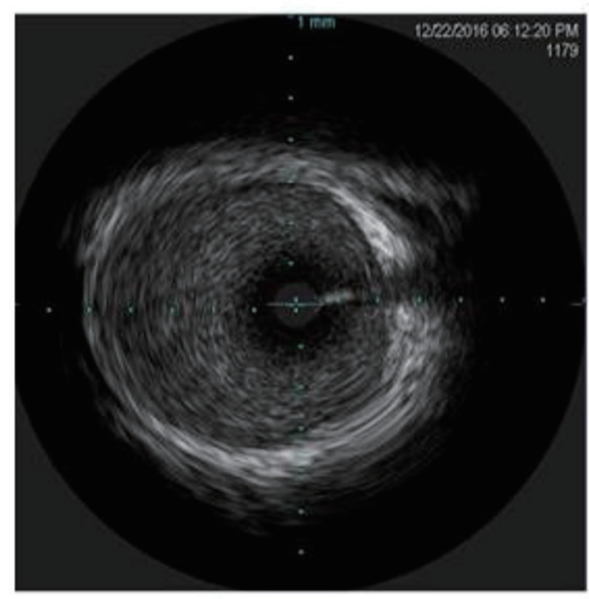

Intramural hematoma resolved

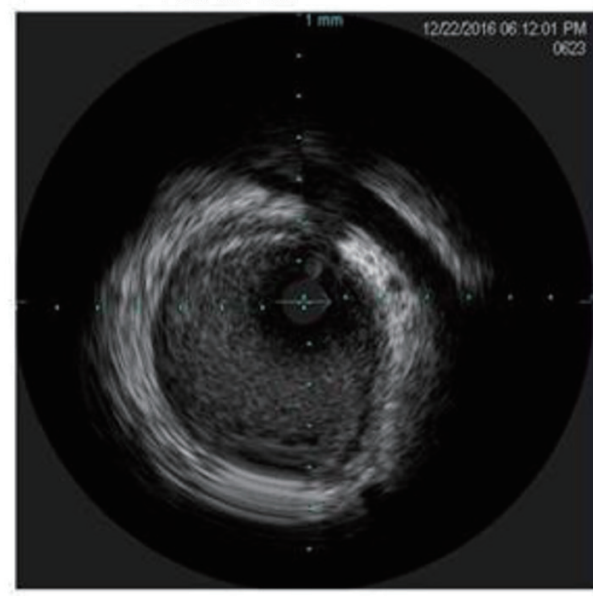

\section{Post-lesion}

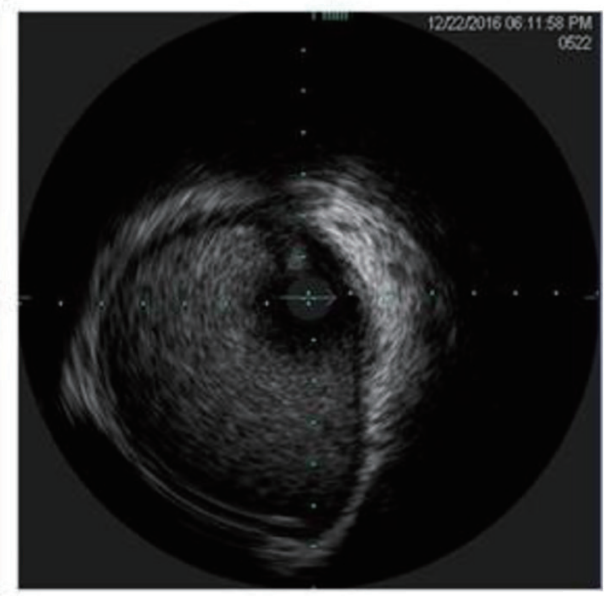

Figure 5. Intravascular ultrasound revealing healed intramural hematoma. 
platelet therapy for future prevention of coronary and cerebral events.

\section{Conclusion}

We report a rare case of SCAD in patient with FMD who presented with SCD as a result of left main coronary artery dissection. The patient was managed successfully with conservative medical therapy. A high index of suspicion for FMD should be maintained in young peripartum female patients presenting with ACS.

\section{References}

1. Mehta LS, Beckie TM, DeVon HA, Grines CL, Krumholz HM, Johnson MN, Lindley KJ, et al. Acute myocardial infarction in women: a scientific statement from the American heart association. Circulation. 2016;133(9):916-947.

2. Amsterdam EA, Wenger NK, Brindis RG, et al. 2014 AHA/ACC guideline for the management of patients with non-ST-elevation acute coronary syndromes: executive summary: a report of the American College of Cardiology/American Heart Association Task Force on Practice Guidelines. J Am Coll Cardiol. 2014;64:2645-2687.

3. Saw J. Spontaneous coronary artery dissection. Can J Cardiol. 2013;29(9):1027-1033.

4. Alfonso F, Paulo M, Lennie V, Dutary J, Bernardo E, Jimenez-Quevedo P, Gonzalo N, et al. Spontaneous coronary artery dissection: long-term follow-up of a large series of patients prospectively managed with a "conservative" therapeutic strategy. JACC Cardiovasc Interv. 2012;5(10):1062-1070.

5. Lettieri C, Zavalloni D, Rossini R, Morici N, Ettori F, Leonzi O, Latib A, et al. Management and long-term prognosis of spontaneous coronary artery dissection. Am J Cardiol. 2015;116(1):66-73.

6. Rogowski S, Maeder MT, Weilenmann D, Haager PK,
Ammann P, Rohner F, Joerg L, et al. Spontaneous coronary artery dissection: angiographic follow-up and long-term clinical outcome in a predominantly medically treated population. Catheter Cardiovasc Interv. 2017;89(1):59-68.

7. Toggweiler S, Puck M, Thalhammer C, Manka R, Wyss M, Bilecen D, Corti R, et al. Associated vascular lesions in patients with spontaneous coronary artery dissection. Swiss Med Wkly. 2012;142:w13538.

8. Olin JW, Gornik HL, Bacharach JM, Biller J, Fine LJ, Gray BH, Gray WA, et al. Fibromuscular dysplasia: state of the science and critical unanswered questions: a scientific statement from the American Heart Association. Circulation. 2014;129(9):1048-1078.

9. Saw J, Aymong E, Sedlak T, Buller CE, Starovoytov A, Ricci D, Robinson S, et al. Spontaneous coronary artery dissection: association with predisposing arteriopathies and precipitating stressors and cardiovascular outcomes. Circ Cardiovasc Interv. 2014;7(5):645-655.

10. Tweet MS, Eleid MF, Best PJ, Lennon RJ, Lerman A, Rihal CS, Holmes DR, Jr., et al. Spontaneous coronary artery dissection: revascularization versus conservative therapy. Circ Cardiovasc Interv. 2014;7(6):777-786.

11. Saw J. Coronary angiogram classification of spontaneous coronary artery dissection. Catheter Cardiovasc Interv. 2014;84(7):1115-1122.

12. Saw J, Mancini GBJ, Humphries KH. Contemporary review on spontaneous coronary artery dissection. J Am Coll Cardiol. 2016;68(3):297-312.

13. Lempereur M, Fung A, Saw J. Stent mal-apposition with resorption of intramural hematoma with spontaneous coronary artery dissection. Cardiovasc Diagn Ther. 2015;5(4):323-329.

14. Epstein AE, DiMarco JP, Ellenbogen KA, Estes NA 3rd, Freedman RA, Gettes LS, Gillinov AM, et al. 2012 ACCF/AHA/HRS focused update incorporated into the ACCF/AHA/HRS 2008 guidelines for device-based therapy of cardiac rhythm abnormalities. J Am Coll Cardiol. 2013;61(3): e6-e75. 